\section{Bioinspired approaches for cancer nanotheranostics}

\author{
"With the recent strides made in the advancement of \\ nanotechnology, many have envisioned this as an innovative \\ component to further empower the field of imaging and \\ diagnostic-based medicine"
}
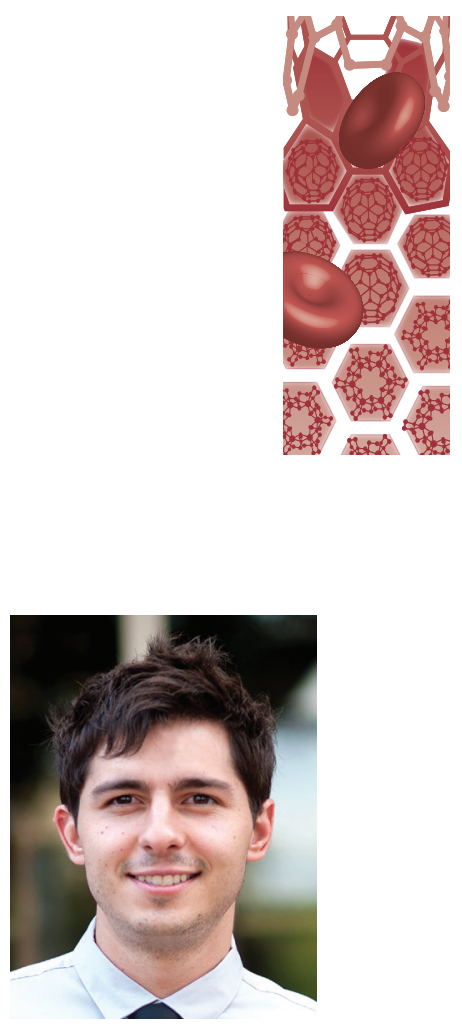

Michael Evangelopoulos Center for Biomimetic Medicine, Houston Methodist Research Institute, Houston, TX 77030, USA progression and response to therapy [1]. With the recent strides made in the advancement of nanotechnology, many have envisioned this as an innovative component to further empower the field of imaging and diagnostic-based medicine [2]. The unique physical and chemical characteristics of nanoparticles (e.g., optical, magnetic, thermal, and imaging properties) have provided unparalleled solutions to diseases, such as localized tumor suppression in colon cancer through the combination of nanoparticle-mediated photodynamic, photothermic and chemo-based therapy [3] or the development of a 'smart' nanoporphyrin platform for the amplification of image sensitivity while providing photothermal therapy [4].

There are currently several theranosticbased approaches that combine therapeutic and diagnostic functions into a single nanoparticle that are either being developed preclinically or entering clinical trials [5]. To wholly harness the capability of these theranostic nanoparticles, scientists have been studying how cells, tissues and organs interact with injected nanomaterials. In our attempt to strategically design a vehicle capable of overcoming the many biological barriers encountered during transport, we decided to leverage on the physiology of the body and, in particular, on the biology of the barriers that nanoparticles must elude. Nonspecific uptake of nanoparticles by healthy organs and sequestration by phagocytic cells are only some of the obstacles that nanoparticles are required to overcome in their journey to diseased tissue [6]. We were among the first to hypothesize and demonstrate that nanoparticles that closely mimic the biological composition of our bodies could have a considerable impact in further maximizing the efficiency of delivery vectors [6-8]. Thus, the development of theranostic technologies that employ biological materials or multi-step delivery vectors could drastically increase the effectiveness of theranostics, leading to a viable one-step treatment solution.

\section{First-generation nanoparticles as imaging modalities}

In their simplest form, nanoparticles have shown unique physiochemical properties for imaging applications. Iron oxide, gold and quantum dot based nanoparticles have all been exploited for the development of modalities that are capable of effectively visualizing and treating cancerous tissue. For example, iron oxide nanoparticles have generated tremendous momentum due to distinctive features such as superparamagnetism, susceptibility to surface modifications (e.g., polyethylene glycol, dextran, polypep-

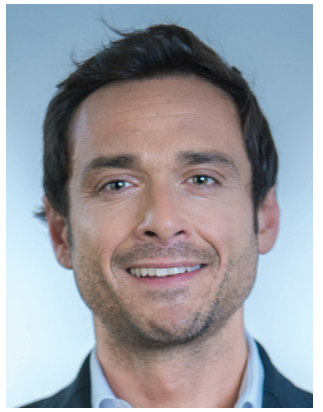

Ennio Tasciotti

Author for correspondence:

Center for Biomimetic Medicine, Houston Methodist Research Institute, Houston, TX 77030, USA and

Department of Orthopedics \& Sports Medicine, Houston Methodist Hospital, Houston, TX 77030, USA

etasciotti@houstonmethodist.org

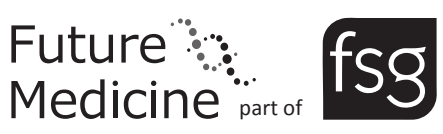


tides) and high surface-to-volume ratios. These features have proven particularly useful for MRI and drug delivery [9]. Gold nanoparticles have also been shown to display similar qualities such as high surface area to volume ratio, as well as cytocompatibility and stability. Additionally, ease of synthesis and the conversion of heat from near-IR light has propelled the use of gold (e.g., nanoshells, nanorods, hollow gold) for a variety of photo-triggered treatments [10]. In one recent example, gold colloids were used to noninvasively detect residual cancer cells and they successfully minimized recurrence in an animal model of microscopic residual disease [11].

"In an environment where nanoparticles are met with a multitude of barriers, developing a defense that can protect them in the journey to their target site is critical.

\section{Implementation of multi-stage delivery strategies}

In the past decade, multi-stage vectors have been demonstrated by us and others as a way to decouple the multitude of tasks required to bypass biological barriers using a nested nanoparticle approach, therefore resulting in increased efficacy. Multi-stage nanovectors have been strategically designed to concentrate drug loading into a single carrier while, concurrently, serving as a shield against the obstacles that nanoparticles typically encounter during circulation. Previously, our group demonstrated that porous silicon particles served as promising theranostic carriers due to the versatile nature of porous silicon and the ability to regulate the release kinetics of a payload, thus resulting in a significant enhancement of $T_{1}$ contrast when primed with gadolinium-based contrast agents [12]. More recent endeavors have demonstrated the exploitation of distinctive environmental factors (e.g., $\mathrm{pH}$, biomolecules, temperature) which can result in significant enhancement of a theranostic payload [13]. Polymer-based vectors designed to swell and contract at designated $\mathrm{pH}$ have exhibited a substantial increase in the localization of therapies, teaching us the importance of rationale design in the development of delivery vectors.

\section{Trend toward bioinspired delivery systems}

More recently, attempts that employ biological materials in the development of nanoparticles have gained increasing attention in overcoming the limitations of drug delivery systems such as biocompatibility, toxicity and targeting. Abraxane (i.e., protein-bound paclitaxel) can be seen as the first bioinspired approach, having been approved more than 10 years ago by the US FDA. Capitalizing on albumin's natural interaction with endothelial cell membrane proteins, Abraxane is admitted with rapid and preferential accumulation at the tumor site, resulting in superior response rates and reduced toxicity compared with conventional paclitaxel. However, the manipulation of biological matter and its incorporation into synthetic carriers has increasingly been explored due to its potential to substantially improve the accumulation of imaging and therapeutic agents. It is for this reason that theranostics based on biological components have been developed to exploit all levels of biological complexity and have demonstrated strongly desirable characteristics as delivery systems.

Lipoprotein-based nanoparticles are one example of a biomimetic approach that has been shown to selectively accumulate conjugated radiolabeled tracers to tumor sites as a result of the extended circulation time granted by lipoproteins. This method of transport has been shown to accommodate a variety of agents and has led to several promising candidates for photodynamic therapy [14]. Similar success has been achieved when drawing inspiration directly from cells within the body $[7,15-17]$. In one instance, the coating of polymeric nanoparticles with erythrocyte-derived cellular membrane, promoted extended circulation, resulting in a small amount of the injected dose still being detected in circulation 3 days post administration [15]. Manipulation of cellular membrane derived from leukocytes displayed comparable benefits in extending particle circulation while simultaneously avoiding nonspecific uptake and demonstrating an active communication with the endothelial barrier [7]. Likewise, the incorporation of leukocyte plasma membranes into lipid nanoparticles maintained all these benefits while providing a significant increase in accumulation to an inflamed site and a reduction in neutrophil infiltration when delivering a therapeutic payload [18]. Further inspiration has been drawn from cell-derived exosomes due to their small size and protein function, despite their relatively unknown composition. Exosomes have been described as transporting biological materials (e.g., proteins, mRNA) to different tissues by utilizing vascular systems [19]. This has led to attempts to isolate the exosomes and load them with a therapeutic payload to exploit their extended circulation time. These factors demonstrate the success that has been achieved in exploited biological material from proportions spanning the entire cell to manipulation of exosome as small as $30 \mathrm{~nm}$.

\section{Looking onward}

In an environment where nanoparticles are met with a multitude of barriers, developing a defense that can protect them in the journey to their target site is critical. Bioinspired and biomimetic delivery platforms have 
demonstrated substantial improvements in the delivery of traditional therapeutic and diagnostic agents. Moving forward, theranostics designed to incorporate biological components traditionally employed by bioinspired approaches could provide unprecedented opportunities in the imaging and treatment of diseases characterized by inflammation. However, to materialize a viable treatment solution through the utilization of biomimetic nanoplatforms, it is important to consider the potential hurdles that may arise. To fully be appreciated in a clinical setting, careful consideration must be given to the components that permit biological formulations to function without unintended consequences. As recently discussed, biological materials often work in a very sophisticated and cohesive manner that may make manipulation of these components not

\section{References}

1 For the first time, neurosurgeons and head and neck surgeons collaborate to utilize surgical theater's 3D planning and navigation systems inside the operating room. www.businesswire.com

2 Weissleder R, Nahrendorf M, Pittet MJ. Imaging macrophages with nanoparticles. Nat. Mater. 13(2), 125-138 (2014).

3 Lee H, Lee Y, Song C et al. An endoscope with integrated transparent bioelectronics and theranostic nanoparticles for colon cancer treatment. Nat. Commun. 6, 10059 (2015).

4 Li Y, Lin TY, Luo Y et al. A smart and versatile theranostic nanomedicine platform based on nanoporphyrin. Nat. Commun. 5, 4712 (2014).

5 ClinicalTrials.gov. Radiosensitization of multiple brain metastases using AGuIX gadolinium based nanoparticles. www.clinicaltrials.gov

6 Blanco E, Shen H, Ferrari M. Principles of nanoparticle design for overcoming biological barriers to drug delivery. Nat. Biotechnol. 33(9), 941-951 (2015).

7 Parodi A, Quattrocchi N, van de Ven AL et al. Synthetic nanoparticles functionalized with biomimetic leukocyte membranes possess cell-like functions. Nat. Nanotechnol. 8(1), 61-68 (2013).

8 Yoo JW, Irvine DJ, Discher DE, Mitragotri S. Bio-inspired, bioengineered and biomimetic drug delivery carriers. Nat. Rev. Drug Discov. 10(7), 521-535 (2011).

9 Laurent S, Forge D, Port M et al. Magnetic iron oxide nanoparticles: synthesis, stabilization, vectorization, physicochemical characterizations, and biological applications. Chem. Rev. 108(6), 2064-2110 (2008).

10 Dykman LA, Khlebtsov NG. Multifunctional gold-based nanocomposites for theranostics. Biomaterials 108, 13-34 (2016). perform as intended [20]. However, recent trends have demonstrated that the development of materials that work synergistically with biologics is feasible, making a personalized theranostic approach to medicine through biomimcry a workable reality.

\section{Financial \& competing interests disclosure}

The authors gratefully acknowledge funding from the George I and Angelina P Kostas Charitable Foundation, William Randolph Hearst Foundation and The Cullen Trust for Health Care. The authors have no other relevant affiliations or financial involvement with any organization or entity with a financial interest in or financial conflict with the subject matter or materials discussed in the manuscript apart from those disclosed.

No writing assistance was utilized in the production of this manuscript.

11 Lukianova-Hleb EY, Kim YS, Belatsarkouski I, Gillenwater AM, O’Neill BE, Lapotko DO. Intraoperative diagnostics and elimination of residual microtumours with plasmonic nanobubbles. Nat. Nanotechnol. 11(6), 525-532 (2016).

12 Ananta JS, Godin B, Sethi R et al. Geometrical confinement of gadolinium-based contrast agents in nanoporous particles enhances $T_{1}$ contrast. Nat. Nanotechnol. 5(11), 815-821 (2010).

13 Caldorera-Moore ME, Liechty WB, Peppas NA. Responsive theranostic systems: integration of diagnostic imaging agents and responsive controlled release drug delivery carriers. Acc. Chem. Res. 44(10), 1061-1070 (2011).

$14 \mathrm{Ng} \mathrm{KK}$, Lovell JF, Zheng G. Lipoprotein-inspired nanoparticles for cancer theranostics. Acc. Chem. Res. 44(10), 1105-1113 (2011).

15 Hu CM, Zhang L, Aryal S, Cheung C, Fang RH, Zhang L. Erythrocyte membrane-camouflaged polymeric nanoparticles as a biomimetic delivery platform. Proc. Natl Acad. Sci. USA 108(27), 10980-10985 (2011).

16 Doshi N, Swiston AJ, Gilbert JB et al. Cell-based drug delivery devices using phagocytosis-resistant backpacks. $A d v$. Mater. 23(12), H105-H109 (2011).

17 Hu CM, Fang RH, Wang KC et al. Nanoparticle biointerfacing by platelet membrane cloaking. Nature 526(7571), 118-121 (2015)

18 Molinaro R, Corbo C, Martinez JO et al. Biomimetic proteolipid vesicles for targeting inflamed tissues. Nat. Mater. 15(9), 1037-1046 (2016).

19 Van Den Boorn JG, Schlee M, Coch C, Hartmann G. SiRNA delivery with exosome nanoparticles. Nat. Biotechnol. 29(4), 325-326 (2011).

20 Dawson KA, Yan Y. Drug delivery: leukocyte-like carriers. Nat. Mater. 15(9), 935-936 (2016). 